\title{
Acetone Bio-sniffer (YSZ-based Electrochemical Gas Phase Biosensor) toward Diagnosis of Diabetes
}

\author{
Xidong Hao ${ }^{1}$, Xishuang Liang*1, Geyu Lu*1 \\ ${ }^{1}$ State Key Laboratory on Integrated Optoelectronics, College of Electronic Science and Engineering, \\ Jilin University, 2699 Qianjin Street, Changchun 130012, China \\ Xishuang Liang, Tel.: +86 431 85168384; fax: +86 43185167808. \\ Email: liangxs@jlu. edu.cn (X. Liang). \\ Geyu Lu, Tel.: +86 431 85167808; fax: +86 43185167808.
}

Email: lugy@jlu. Edu.cn (G. Lu).

\begin{abstract}
:
Acetone bio-sniffer, because of its ability of continuous non-invasive monitoring, is recognized as a potential method for the diagnosis of diabetes. In this study, mixed potential electrochemical sensors based on $\mathrm{YSZ}$ and $\mathrm{K}_{2} \mathrm{NiF}_{4}$-type oxides $\mathrm{Sm}_{2-x} \mathrm{Sr}_{x} \mathrm{NiO}_{4}(\mathrm{x}=0.4,0.6$ and 0.8$)$ sensing electrode were fabricated as bio-sniffer for diagnosis of diabetics by detecting acetone concentration in exhaled breath. The results showed that when $\mathrm{Sm}_{1.4} \mathrm{Sr}_{0.6} \mathrm{NiO}_{4}$ was used as sensing material, the fabricated sensor exhibited the best performance in comparison with other sensors, the present device also exhibited prominent reliability, excellent humidity resistance and good stability over 30 days. What's more, the low detection limit of sensor to acetone was $300 \mathrm{ppb}$, indicating that the sensor had ability for acetone detection in exhaled breath. The exhaled breathes of the diabetics with ketosis were used for detection and results showed that the sensor had a manifest and stable signal. Besides, the response and recovery time were also acceptable to real-time detection. In addition, the relationship of the blood ketone level and the acetone concentration in exhaled breath was given in the paper. Above all, the fabricated sensor has enormous potentiality for the diabetes monitoring through breath analysis.
\end{abstract}

Key words: Bio-sniffer, Acetone, Mixed potential electrochemical sensor, Exhaled breath

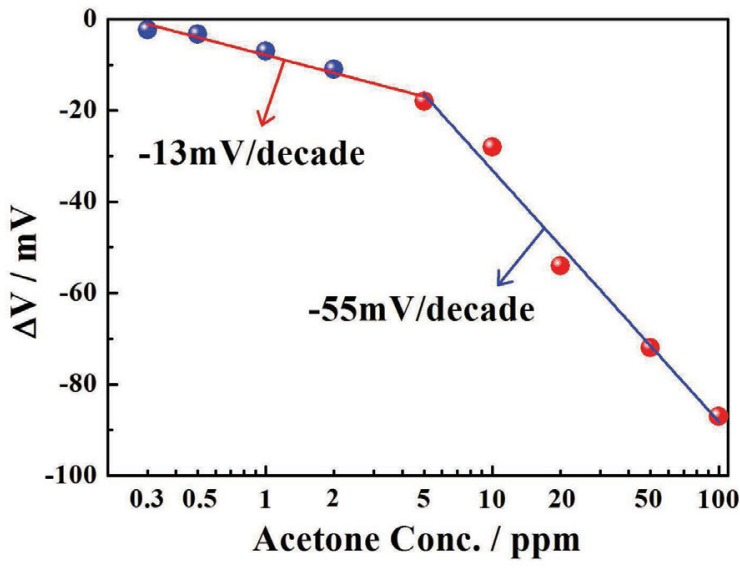

Fig. 1. Dependence of $\Delta V$ for the sensor attached with $\mathrm{Sm}_{1.4} \mathrm{Sr}_{0.6} \mathrm{NiO}_{4}$-SE on logarithm of acetone concentration in the range of $0.3-100 \mathrm{ppm}$ at $675^{\circ} \mathrm{C}$.

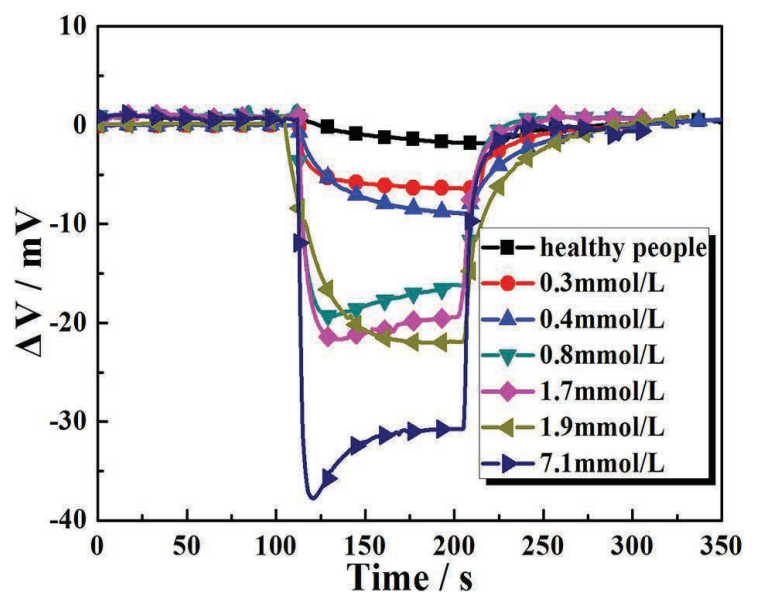

Fig. 2. The response and recovery transient curves of sensor to the volunteer of different blood ketone levels.

\section{References}

[1] ATH Risby, FK Tittel, Current status of midinfrared quantum and interband cascade lasers for clinical breath analysis, Optical Engineering, 201049 (11) 792-796. 
[2] B. Wang, F. Liu, X. Yang, Y. Guan, C. Ma, X. Hao, X. Liang, F. Liu, P. Sun, T. Zhang, and G. $\mathrm{Lu}$, Fabrication of Well-Ordered Three-Phase Boundary with Nanostructure Pore Array for Mixed Potential-Type Zirconia-Based $\mathrm{NO}_{2}$ Sensor, ACS Appl. Mater. Interfaces. 8 (2016) 1675216760.

[3] C. Wang, A. Mbi, M. Shepherd, A study on breath acetone in diabetic patient using a cavity ringdown breath analyzer; Exploring correlations of breath acetone with blood glucose and glycohemoglobin AIC, Sensors Journal, IEEE, 201010 (1) 54-63.

[4] Corrado Di Natale, Antonella Macagnano, Eugenio Martinelli,Roberto Paolesse, Giuseppe D'Arcangelo, Claudio Roscioni, Alessandro Finazzi-Agro, Arnaldo D'Amico, Lung cancer identification by the analysis of breath by means of an array of non-selective gas sensor, Biosensors and Bioelectronics 18 (2003) 12091218.

[5] D. Guo, D. Zhang, L. Zhang, G. Lu, Non-invasive blood glucose monitoring for diabetics by means of breath signal analysis, Sens. Actuators B 173 (2012) 160-113.

[6] N. Miura, J. Wang, M. Nakatou, P. Elumalai, S. Zhuiykov, M. Hasei, High-temperature operating characteristics of mixed-potential-type $\mathrm{NO}_{2}$ sensor based on stabilized-zirconia tube and $\mathrm{NiO}$ sensing electrode, Sens. Actuators B: Chem. 114 (2006) 903-909.

[7] N. Miura, J. Wang, M. Nakatou, P. Elumalai, S. Zhuiykov, M. Hasei, High-temperature operating characteristics of mixed-potential-type $\mathrm{NO}_{2}$ sensor based on stabilized-zirconia tube and $\mathrm{NiO}$ sensing electrode, Sens. Actuators B: Chem. 114 (2006) 903-909.

[8] T. Xiao, X. Wang, Z. Zhao, L. Li, L. Zhang, H. Yao, J. Wang, Z. Li, Highly sensitive and selective acetone sensor based on C-doped $\mathrm{WO}_{3}$ for potential diagnosis of diabetes mellitus, Sens. Actuators B: Chem. 199 (2014) 210-219.

[9] V. N. Chaudhari, A. P. Khandale, S. S. Bhoga, An investigation on strontium doped $\mathrm{Sm}_{2} \mathrm{NiO}_{4-} \delta$ cathode for intermediate temperature solid oxide fuel cells. Journal of Power Sources 248 (2014) 647-654.

[10] Van den Velde, S., Nevens, F., Van, H. P. , van, S. D., Quirynen, M., $2008 . \quad J o u r n a l$ of Chromatography. B, Analytical Technologies in the Biomedical and Life Sciences 875, 344-348.

[11] W. Li, Y. Liu, X. Lu, et al, A cross-sectional study of breath acetone based on diabetic metabolic disorders, J Breath Res, 20149 (1) 016005.

[12] W. Chuji, S. Peeyush, Breath analysisi using laser spectroscopic techniques:breath biomarkers, spectral fingerprints, and detection limits, Sensors (Baesl), 2009, 9 (10) 8230-62.

[13] X. D. Hao, B. Wang, C. Ma, F. M. Liu, X. Yang, T. Liu, X. S. Liang, C. H. Yang, H. Q. Zhu, G. Y. Lu, Mixed potential type sensor based on stabilized zirconia and $\mathrm{Co}_{1-x} \mathrm{Zn}_{\mathrm{x}} \mathrm{Fe}_{2} \mathrm{O}_{4}$ sensing electrode for detection of acetone, Sensors and Actuators B 255 (2018) 1173-1181.

[14] Y. Chen, J. Z. Xiao, Effects of YSZ addition on the response of $\mathrm{La}_{2} \mathrm{CuO}_{4}$ sensing electrode for a potentiometric $\mathrm{NO}_{x}$ sensor, Sens. Actuators B: Chem. 192 (2014) 730-736.

[15] Yael Zilberman, Sameer R. Sonkusale, Microfluidic optoelectronic sensor for salivary diagnostics of stomach cancer, Biosensors and Bioelectronics 67 (2015) 465-471. 